\title{
DERIVATION OF THE HOPF-COLE SOLUTION TO BURGERS' EQUATION BY STOCHASTIC INTEGRALS ${ }^{1}$
}

\section{S. I. ROSENCRANS}

ABSTRACT. In this paper the Hopf-Cole solution to Burgers' equation is derived by use of stochastic integrals. First the equation is written in Hamilton-Jacobi form, and then, following an idea of Freidlin, the solution is differentiated along a Brownian motion.

Let $u$ be a solution to the (backwards) Cauchy problem for Burgers' equation

$$
\begin{aligned}
u_{t}+u u_{x}+\frac{1}{2} u_{x x} & =0, \quad t<T, x \in \boldsymbol{R}, \\
u(T, x) & =g(x),
\end{aligned}
$$

where $g$ is bounded and measurable. In conservation form, the equation becomes

$$
u_{t}+\frac{1}{2}\left(u^{2}+u_{x}\right)_{x}=0 .
$$

We make the change of variables $u=\phi_{x}$; the motivation for considering $\phi$ comes from Hamilton-Jacobi theory. Then the equation can be integrated with respect to $x$ to yield

$$
\begin{aligned}
\phi_{t}+\frac{1}{2} \phi_{x}^{2}+\frac{1}{2} \phi_{x x} & =0, \quad t<T, x \in \boldsymbol{R}, \\
\phi(T, x) & =G(x),
\end{aligned}
$$

where $G^{\prime}=g$. (Here we have if necessary added to $\phi$ an appropriate function of time so that the equation is rendered homogeneous.) We now consider the stochastic differential of $\phi$ along the diffusion governed by the linear part of the operator, i.e., the Brownian motion

$$
y_{r}=z+b_{r}-b_{s}, \quad s \leqq r \leqq T,
$$

where $b$ is a standard Brownian motion starting at zero and $z \in R$ and $s<T$ are fixed. Then by Ito's lemma [1, p. 32]

Presented to the Society, April 10, 1971 under the title A probabilistic derivation of the Hopf-Cole solution to Burgers' equation; received by the editors April 19, 1971.

AMS 1970 subject classifications. Primary 35Q99, 35K55; Secondary 60H05.

Key words and phrases. Burgers' equation, stochastic integral, Ito's lemma.

1 This research was supported in part by NSF Contract GP-19824. 


$$
\begin{array}{rlrl}
d \phi\left(r, y_{r}\right) & =\phi_{r} d r+\phi_{x} d y_{r}+\frac{1}{2} \phi_{x x}\left(d y_{r}\right)^{2} & \\
& =\phi_{r} d r+\phi_{x} d b_{r}+\frac{1}{2} \phi_{x x} d r & \\
& =\phi_{x} d b_{r}-\frac{1}{2} \phi_{x}^{2} d r, \quad s<r<T .
\end{array}
$$

We recognize the right-hand side as the differential occuring in the integrand of the exponential martingale [1, p. 25]

$$
Z(t)=\exp \left\{\int_{s}^{t} \phi_{x} d b_{r}-\frac{1}{2} \int_{s}^{t} \phi_{x}^{2} d r\right\} .
$$

(In general $Z$ would be only a supermartingale, but from the maximum principle we know $\phi_{x}$ is bounded.) This suggests integrating (1) over $(s, T)$ :

$$
\phi\left(T, y_{T}\right)-\phi\left(s, y_{s}\right)=\log Z(T),
$$

and then taking the exponential of both sides,

$$
\exp \left[\phi\left(T, y_{T}\right)-\phi\left(s, y_{s}\right)\right]=Z(T) .
$$

Note that $y_{s} \equiv z$ and $\phi(T, x)=G(x)$, hence this becomes

$$
\exp \left[G\left(y_{T}\right)-\phi(s, z)\right]=Z(T) .
$$

But since $Z$ is a martingale the expectation of $Z(t)$ is constant and equal to unity, hence

$$
. \exp [\phi(s, z)]=E \exp \left[G\left(y_{T}\right)\right] .
$$

Next take the logarithm of both sides and differentiate with respect to $z$ :

$$
u(s, z)=\frac{\partial}{\partial z} \log E \exp \left[G\left(y_{T}\right)\right]=\frac{\partial}{\partial z} \log H_{T-s} \exp [G(z)]
$$

where $H_{t}$ stands for convolution with the heat kernel at time $t$. This is the Hopf-Cole solution. (See [2].)

For completeness we should mention another use of the martingale $Z$. It is easy to show, by considering the differential of $t \rightarrow u\left(t, y_{t}\right) Z(t)$ that

$$
u(s, z)=E\left[g\left(y_{T}\right) Z(T)\right], \quad s<T .
$$

(This is actually a form of the generalized Cameron-Martin formula. See [1, p. 67].) Representations such as this were used by Freidlin [3] to prove existence of a global solution to a wide class of quasilinear Cauchy problems.

In a direction converse to that in this note, Henry McKean has started with the Hopf-Cole solution and given it a probabilistic interpretation. This work is unpublished. 


\section{REFERENCES}

1. H. P. McKean, Jr., Stochastic integrals, Probability and Math. Statist., no. 5, Academic Press, New York, 1969. MR 40 \#947.

2. E. Hopf, The partial differential equation $u_{t}+u u_{x}=\mu u_{x x}$, Comm. Pure Appl. Math. 3 (1950), 201-230. MR 13, 846.

3. M. I. Freĭdlin, Quasilinear parabolic equations, and measures in a function space, Funkcional. Anal. i Priložen. 1 (1967), no. 3, 74-82. MR 37 \#584.

Department of Mathematics, Tulane University, New Orleans, Louisiana 70118

Current address: Department of Mathematics and Statistics, University of New Mexico, Albuquerque, New Mexico 87106 